\title{
Opportunities and limits of the one gene approach: the ability of Atoh1 to differentiate and maintain hair cells depends on the molecular context
}

\author{
Israt Jahan, Ning Pan and Bernd Fritzsch*
}

Department of Biology, University of lowa, lowa City, IA, USA

Edited by:

Andy Groves, Baylor College of

Medicine, USA

\section{Reviewed by:}

Thomas Schimmang, Consejo

Superior de Investigaciones

Cientificas, Spain

Albert Edge, Harvard Medical

School, USA

\section{*Correspondence.}

Bernd Fritzsch, Department of

Biology, College of Liberal Arts and

Sciences, University of lowa, 143

Biology Building, 129 E Jefferson

Street, lowa City, IA 52242-1324,

USA

e-mail: bernd-fritzsch@uiowa.edu
Atoh1 (Math1) was the first gene discovered in ear development that showed no hair cell $(\mathrm{HC})$ differentiation when absent and could induce $\mathrm{HC}$ differentiation when misexpressed. These data implied that Atoh1 was both necessary and sufficient for hair cell development. However, other gene mutations also result in loss of initially forming HCs, notably null mutants for Pou4f3, Barh/1, and Gfi1. HC development and maintenance also depend on the expression of other genes (Sox2, Eya1, Gata3, Pax2) and several genes have been identified that can induce HCs when misexpressed (Jag1) or knocked out (Lmo4). In the ear Atoh 1 is not only expressed in HCs but also in some supporting cells and neurons that do not differentiate into HCs. Simple removal of one gene, Neurod1, can de-repress Atoh1 and turns those neurons into HCs suggesting that Neurod1 blocks Atoh1 function in neurons. Atoh 1 expression in inner pillar cells may also be blocked by too many Hes/Hey factors but conversion into HCs has only partially been achieved through Hes/Hey removal. Detailed analysis of cell cycle exit confirmed an apex to base cell cycle exit progression of $\mathrm{HCs}$ of the organ of Corti. In contrast, Atoh1 expression progresses from the base toward the apex with a variable delay relative to the cell cycle exit. Most HCs exit the cell cycle and are thus defined as precursors before Atoh 1 is expressed. Atoh 1 is a potent differentiation factor but can differentiate and maintain HCs only in the ear and when other factors are co-expressed. Upstream factors are essential to regulate Atoh1 level of expression duration while downstream, co-activated by other factors, will define the context of Atoh1 action. We suggest that these insights need to be taken into consideration and approaches beyond the simple Atoh 1 expression need to be designed able to generate the radial and longitudinal variations in hair cell types for normal function of the organ of Corti.

Keywords: Atoh1, hair cells, development, regeneration

\section{INTRODUCTION}

The idea that single genes might be responsible for hair cell (HC) development and thus could be used to regenerate HCs and restore hearing was born in the late 1990s: Mice with a deletion of the Pou domain gene Pou4f3 (aka Brn3c, Brn3.1) were completely deaf, "owing to a failure of HCs to appear in the inner ear, with subsequent loss of cochlear and vestibular ganglia" (Erkman et al., 1996). This mouse mutant derived conclusion was soon followed by data on human mutations showing that a truncating mutation of the human POU4f3 gene is the basis of DFNA15, resulting in progressive hearing loss (Vahava et al., 1998). Subsequent work showed that HCs initially form and develop normal in Pouff3 mutants, but eventually die in a base to apex progression (Xiang et al., 2003; Hertzano et al., 2004). While the initial work claimed loss of all sensory neurons, later work showed that some neurons remain for 6 months in a dedifferentiated organ of Corti (OC) that shows Atoh1-lacZ and Myo7a positive cells (Pauley et al., 2008). The original claim of "failure of HCs to appear" was thus transformed into a rather normal initial development followed by HC death. Pou 4 f3 is now recognized as a maintenance factor for HCs, like Gfi1 and Barhl1 (Li et al., 2002; Hertzano et al., 2004) that is expressed in adult HCs through complex regulation, including possibly the bHLH gene Atoh1 (Ahmed et al., 2012; Masuda et al., 2012).

Why is this background information on Pou4f3 relevant for the discussion of the role of Atoh1 (aka Math1) for HC differentiation and maintenance? In the following we will explore that Atoh1 has much in common with Pou4f3 in terms of claims raised as a gene that is "necessary and sufficient" for HC differentiation (Chen et al., 2002; Giraldez and Fritzsch, 2007; Groves et al., 2013). In contrast to this claim, the millions of neurons outside the ear expressing Atoh1 (Mulvaney and Dabdoub, 2012) never turn into HCs, suggesting that Atoh1 is not sufficient to induce HCs everywhere where Atohl is expressed. Only the molecularly unclear context of certain cells of the ear allows Atoh1 to drive HC differentiation and maintenance. Even in the ear, Atoh1 is expressed in many cells (Matei et al., 2005) that require additional manipulations to turn into HCs (Jahan et al., 2010), indicating that expression of Atoh1 in the ear does not guarantee differentiation of all cells into HCs. As with Pou4f3, 
it appears that Atoh1 absence is compatible with some cellular differentiation, indicating that Atoh1 is not defining HCs but is differentiating them (Jahan et al., 2012). The delayed and profound loss of HCs in a "self-terminating" Atoh1 system (Pan et al., 2012) and hypomorphic Atoh1 mutant (Sheykholeslami et al., 2013) suggests an essential role in maintenance, possibly including adult expression of Pou4f3 (Masuda et al., 2012). Consistent with Atoh1 being an essential differentiation and maintenance factor for $\mathrm{HC}$ is the fact that overexpression can rescue HCs (Yang et al., 2012). Like Pou4f3, Atoh1 is necessary to differentiate and maintain HCs. It remains to be shown whether forced expression of Atoh1 (Kelly et al., 2012) can differentiate HCs when certain factors are absent (Zou et al., 2004; Kiernan et al., 2005; Bouchard et al., 2010; Ahmed et al., 2012; Duncan and Fritzsch, 2013; Schimmang, 2013) that define the context for Atoh1 action in the ear thus providing the competency to respond to Atoh1 protein. Below we explore some issues related to Atoh1 function that remain underexplored in many contemporary reviews and propose novel strategies to maintain HCs.

\section{EXPRESSION OF Atoh1 OUTSIDE THE EAR DOES NOT LEAD TO HC DIFFERENTIATION}

Atoh1 was isolated from cerebellar granule cells, the largest population of neurons in the human brain, amounting to over 60 billion neurons (Ben-Arie et al., 1997; Herculano-Houzel, 2009). Atoh1 is expressed in the proliferative precursor population of the external granule cell layer where it is needed to generate the billions of granule cells (Pan et al., 2009). Atoh1 is also essential for medulloblastoma progression and Atoh1 removal reduces the progression of this childhood tumor (Flora et al., 2009). In contrast to this expression of Atoh1 in proliferating precursors in the CNS, the expression of Atoh1 in the mouse cochlea is predominantly in post-mitotic HCs, with a possible overlap of Atoh1 expression and cell cycle exit in the basal turn HCs (Ruben, 1967; Matei et al., 2005; Lee et al., 2006). A pulse-chase experiment using BrdU or EdU labeling followed by in situ hybridization for Atoh1 around E14 is needed to verify this suggestion of possible Atohl expression in proliferating $\mathrm{HC}$ precursors. In the apex there is no expression of Atoh1 prior to cell cycle exit, indicating that HC precursor specification and cell cycle exit is independent of Atoh1 (Jahan et al., 2013; Kopecky et al., 2013). Both premature expression of Atoh1 in Neurod1 null mutants (Jahan et al., 2010) or delayed expression of Atoh1 in Lmx1a null mice (Nichols et al., 2008) results in aberrant development of HCs, implying that onset and level of expression of Atoh1 is tightly regulated to ensure normal differentiation of the right $\mathrm{HC}$ type at the right place (Jahan et al., 2013). Importantly, forced expression of Atoh1 can in postnatal mice induce supporting cell conversion (Liu et al., 2014a) and induces proliferation (Kelly et al., 2012), showing that under these forced conditions Atoh1 exerts functions beyond its tightly regulated function in the embryonic ear. In summary, one of the conditions in which Atoh1 expression in the ear differs from other systems is its expression presumably exclusively in post-mitotic undifferentiated HC precursors whereas in other developing systems Atoh1 is primarily expressed in proliferating precursors.

\section{UPSTREAM AND DOWNSTREAM INTERACTIONS OF Atoh1}

Before Atoh1 can differentiate post-mitotic HC precursors into HCs, the HC precursors have to be specified in the right place and have to receive a signal to exit the cell cycle. Numerous TFs have been identified that are expressed prior to Atohl and affect HC differentiation. For example, Sox2 hypomorphic mice (Kiernan et al., 2005), Pax2 null mice (Bouchard et al., 2010), Eya1 null mice (Zou et al., 2004), and Gata3 conditional null mice (Duncan and Fritzsch, 2013) all show no differentiation of HCs in the cochlea duct but may show variable development of some vestibular HCs, suggesting a unique combinatorial requirement of these genes for cochlear HC development. Misexpression of Jag1 (Pan et al., 2010) or Sox2 (Pan et al., 2013) as well as loss of Lmo4 (Deng et al., 2014) can induce ectopic formation of HCs. In particular work on Eya1/Six1 showed that Atoh1 is but an essential link in a succession of decision making steps (Ahmed et al., 2012) toward HC differentiation (Figure 1) with unknown regulatory complexity.

Atoh1 regulates the expression of hundreds of downstream genes (Klisch et al., 2011). Some of these genes are TFs that in turn regulate expression of several hundred downstream genes. One of the TFs that are regulated by Atoh1, is Neurod1. Atoh1 is in a positive autoregulatory loop whereby Atoh1 stimulates its own expression through an enhancer sequence (Figure 1). Such loops are typically counterbalanced by negative feedback to ensure upper limits of expression. Neurod1 is part of this negative feedback loop and controls the level of Atoh1 expression in developing systems such as the cerebellum (Pan et al., 2009), the intestine (Itkin-Ansari et al., 2005), and the ear (Jahan et al., 2010, 2013). Absence of Neurod1 causes prolonged expression of Atoh1 in precursor cells (external granule cell layer) of the cerebellum that are unable to migrate and differentiate and eventually die (Pan et al., 2009). In the ear, absence of Neurod1 causes transformation of sensory neurons into HCs through disinhibition of a transient Atoh1 in neurons (Jahan et al., 2010) and disruption of the patterning of the $\mathrm{OC}$ by altering the $\mathrm{HC}$ and supporting cell types (Jahan et al., 2013). Some regulation of Atoh1 is also reported in mutants of Hes1/5 (Zine et al., 2001, 2014) and Hey1/2 (Benito-Gonzalez and Doetzlhofer, 2014) but results only in additional formation of HCs outside the OC with limited effects on the patterning of HCs and supporting cells in the OC. Atoh1 is not only regulating the expression of downstream genes but also suppresses upstream genes such as Sox2 (Figure 1). In fact, downregulation of Sox2 appears to be a crucial step for the transition from HC precursors to differentiated HCs (Dabdoub et al., 2008) in agreement with many other differentiating neurosensory system (Reiprich and Wegner, 2014).

Combined, these data show that the early implications of Atohl as the "sole" factor necessary and sufficient to make HCs have to be adjusted to accommodate the emerging concept of Atoh1 integration into a gene network that allows a coordinated transition from the placodal stage to the fully differentiated $\mathrm{HC}$ (Ahmed et al., 2012). Arguably, Atoh1 is enabling a very essential step in this progression toward a $\mathrm{HC}$, but is apparently not needed for precursors to exit the cell cycle and to initiate $\mathrm{HC}$ differentiation (Jahan et al., 2012). However, Atoh1 is a key to HC differentiation (Kelly et al., 2012) and its continued expression 


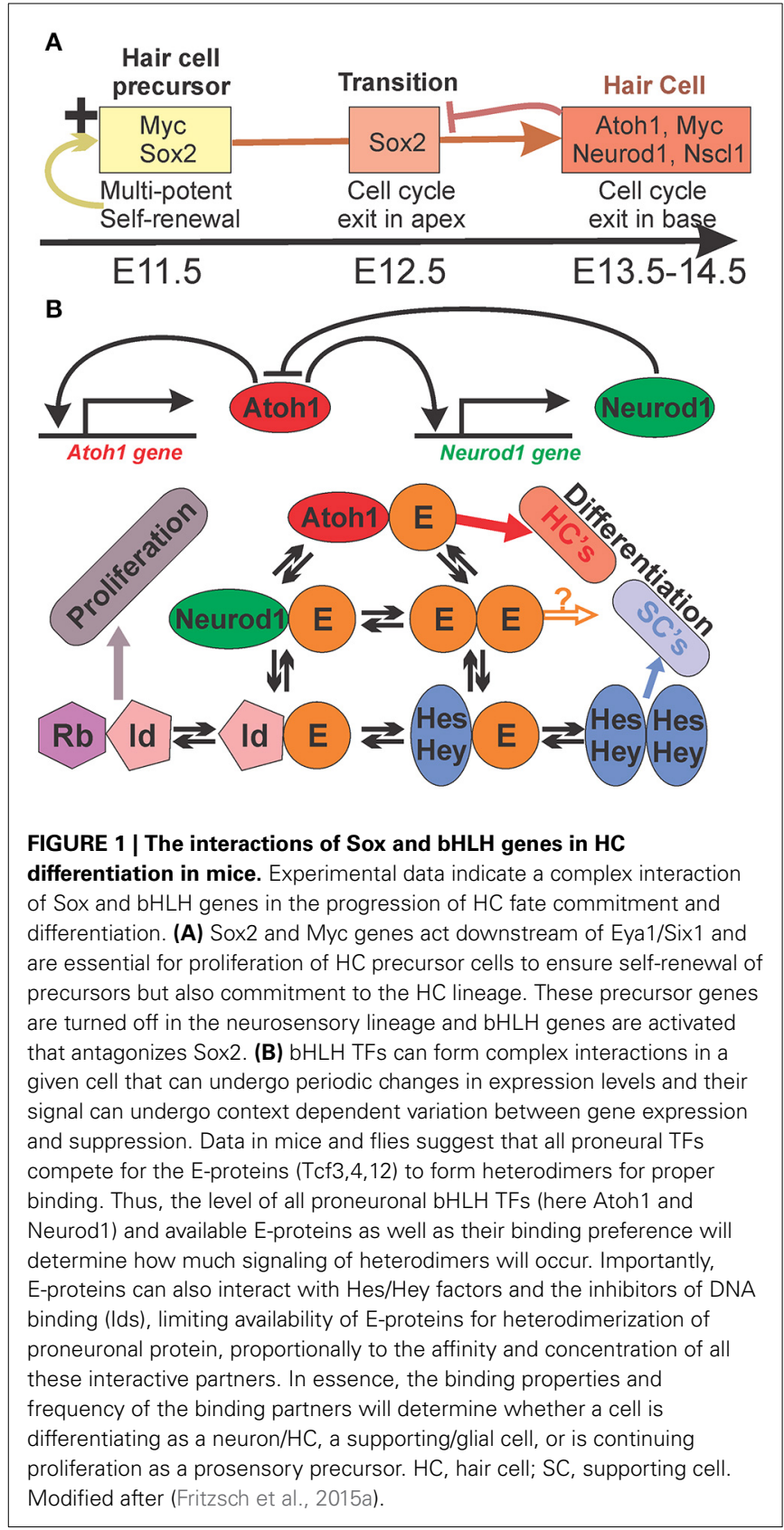

may be essential to maintain differentiated HCs through expression of other genes such as Gfi1, Pou4f3, and Barhl1 (Masuda et al., 2012).

\section{CELL CYCLE EXIT AND Atoh1 EXPRESSION}

Proliferating neurosensory precursor cells are characterized by the expression of multiple transcription factors (TFs) (Ono et al., 2014) and manipulating cell cycle regulation can result in increased (Mantela et al., 2005; Schimmang and Pirvola, 2013) or decreased HCs (Kopecky et al., 2011, 2013). Together these factors ensure that proliferating HC precursors retain a neurosensory determination but continue proliferation to generate more neurosensory cells, under certain conditions and in certain species as stem cells throughout life, like in the olfactory system (Gokoffski et al., 2011). Nearly ubiquitous in these stem cells is the expression of Sry-box gene Sox2 (Reiprich and Wegner, 2014) and several Helix-loop-Helix (HLH) genes (Figure 1), in particular Hes, N-Myc and ID genes, but also some proneural basic HelixLoop-Helix (bHLH genes) such as Ascl1, Neurog1 and, rarely, Atoh1 (Ma et al., 1996, 1998; Imayoshi and Kageyama, 2014). Sox genes and bHLH genes are each engaged in a complicated interaction with members of their own class of genes within a given precursor (Fritzsch et al., 2006; Imayoshi and Kageyama, 2014; Reiprich and Wegner, 2014) but also show cell-cell interactions through Delta-Notch mediated regulation of bHLH genes between cells (Benito-Gonzalez and Doetzlhofer, 2014). In particular, the intracellular interactions established through intrinsic and extrinsic signal mediated fluctuation of expression levels is the basis for a coordinated transition between precursors and differentiated cells (Figure 1). How HC precursors are specified in the right topology of the OC, how the cell cycle exit of HC precursors is regulated and exactly when precursors are committed to $\mathrm{HC}$ differentiation by which molecular means remains an open question despite recent insights into the regulation (Ahmed et al., 2012; Masuda et al., 2012). Among bHLH genes, Myc genes are playing a major role in regulating the numbers of $\mathrm{HCs}$ (Domínguez-Frutos et al., 2011; Kopecky et al., 2011) but are later also expressed in adult HCs where they play no discernable function (Kopecky et al., 2012). We will here explore only the role of proneural bHLH genes in this process, also other TFs undoubtedly play a role in $\mathrm{HC}$ specification and proliferation (Kiernan et al., 2005; Dabdoub et al., 2008; Rocha-Sanchez et al., 2011; Ahmed et al., 2012; Schimmang and Pirvola, 2013).

\section{LOSING Atoh1 AT DIFFERENT STAGES RESULTS IN DIFFERENT EFFECTS}

In the original paper describing absence of HC differentiation in Atoh1 null mutant mice, some supporting cells stain for the LacZ used to replace Atoh1 (Bermingham et al., 1999). A follow up study using an Atoh1 enhancer element to drive fluorescent GFP (Chen et al., 2002) showed that Atoh1 is only expressed in post-mitotic cells to drive their differentiation. In addition, degenerative cells were found in the OC of Atoh1 null mice, suggesting that the primordial HCs form independently of Atoh1 but degenerate without Atoh1. Both papers indicated one major difference: Atoh1-LacZ expression in supporting cells of Atoh1 null mutants whereas no such misexpression was reported using GFP. A subsequent paper using the LacZ insertion (Woods et al., 2004) claimed an initial widespread expression at E13.5. This paper did not correlate the apparent absence of Atoh 1 expression in the apex with the HC cycle exit known to start in the apex (Ruben, 1967; Fritzsch and Nichols, 1993).

Using the same LacZ knockin model as previous papers (Bermingham et al., 1999; Woods et al., 2004), a follow up paper on homozygotic Atoh1-LacZ mice showed continued presence of a single row of undifferentiated LacZ positive cells (Fritzsch et al., 2005) which were spared by the otherwise prevalent apoptosis of most HC precursors (Chen et al., 2002). Subsequent work demonstrated that fluorescent GFP marker (Chen et al., 2002) appeared in nearly every inner pillar cell (Matei et al., 2005; 
Fritzsch et al., 2015b). Furthermore, a novel mouse line using the same enhancer element to drive Cre showed similar expression of Atoh1 in many inner pillar cells (Matei et al., 2005). These data implied, but did not proof beyond doubt that Atoh1 was expressed in inner pillar cells and inferred that the remaining Atoh1-LacZ positive cells in mutants were indeed supporting cells (possibly inner pillar cells) as originally claimed (Bermingham et al., 1999). Further work using a conditional approach to eliminate Atoh1 resulted in nearly identical data, implying that the surviving cells in the absence of Atoh1 might indeed be inner pillar cells in the OC (Pan et al., 2011). Additional work has meanwhile confirmed with different techniques that Atoh1 is indeed prominently expressed in inner pillar cells (Driver et al., 2013). Atoh1 expression in inner pillar cells may be counterbalanced by Hes and Hey factors (Doetzlhofer et al., 2009) and a subsequent paper showed occasional conversion of inner pillar cells to HCs (Benito-Gonzalez and Doetzlhofer, 2014). Atoh1 expression has also been reported in delaminating sensory neurons (Matei et al., 2005) and elimination of Neurod 1 suffices to turn some neurons into HCs expressing Atoh1 and Myo7a (Jahan et al., 2010). Combined, these data suggest that Atoh1 expression alone does not suffice to turn just any cell in the ear into a $\mathrm{HC}$ as co-expressed factors may inhibit this. At least inner pillar cells may be able to survive without Atoh1 protein while maintaining LacZ expression of the Atoh1 locus (Matei et al., 2005; Driver et al., 2013) and are not transformed to HCs even under forced ubiquitous expression of Atoh1 (Kelly et al., 2012).

More recent data provide yet a more complicated picture of lack of Atoh1 expression on HC and OC differentiation. Using an Atoh1 enhancer to drive Cre that activates the Cre only upon presence of Atoh1 protein combined with floxed Atoh 1 generates a "self-terminating" system that results in loss of Atoh1 after a transient presence of Atoh1 protein (Pan et al., 2012). The level of Atoh1 protein depends on the speed with which the Cre can excise the floxed Atoh1 and how long residual Atoh1 protein remains in the cell. Thus, while all cells will see recombination of the LoxP flanked Atoh1, this varies between HCs and thus results in different delay lines of HC precursor apoptosis (Pan et al., 2012). While many HC precursors die rapidly, others survive for several days. Moreover, stretches of the first row of outer HCs survive adjacent to well differentiated inner pillar cells indicating an unusual difference in susceptibility between inner and outer HCs as well as within HC rows in a base to apical gradient. This conclusion is also supported by transgenic knockin mouse where Atoh1 is replaced by Neurog1 (Jahan et al., 2012) which shows that some HC precursors can survive without ever expressing Atoh1. A recently available hypomorph mutant of Atoh1 shows a somewhat similar picture of longitudinal and a less clear radial HC loss (Sheykholeslami et al., 2013) indicating that Atoh1 needs to be present at a critical level to assure long term $\mathrm{HC}$ viability.

Data using inducible Cre expression have complicated this picture even further by showing a rapid and complete loss of all HCs when Cre is induced at different stages of late development (Cai et al., 2013; Chonko et al., 2013). Some claims about abortive transdifferentiation of supporting cells into HCs (Cai et al., 2013) need to be considered in the context of Atoh1 expression in one specific type of supporting cell, the inner pillar cell (Matei et al.,
2005; Driver et al., 2013; Fritzsch et al., 2015b). Despite these minor discrepancies, all papers confirm earlier work and demonstrate that Atoh1 expression is needed to mature and maintain HCs.

In summary, Atoh1 is, much like Pou4f3, a critical factor for $\mathrm{HC}$ differentiation and long term maintenance. Atoh1 is involved in regulating Pou4f3 whereas and its long term expression may be dependent on Atoh1 expression. Further work combining the recently reported hypomorphic allele (Sheykholeslami et al., 2013) with conditional deletion of a floxed Atoh 1 allele (Pan et al., 2012) could detail how level of Atoh1 expression and duration combine for normal $\mathrm{HC}$ maturation and maintenance.

\section{SUMMARY AND OUTLOOK}

Why is it important to go beyond the idea of "necessary and sufficient" for Atoh1 function in the ear? First, while unregulated expression of Atoh1 can convert most ear cells into hair cells (Kelly et al., 2012), nobody has been able to regenerate the two types of HCs that are essential for normal OC function in the right proportion and the right distribution to ensure function (Beurg et al., 2014). In fact, our limited insights into the molecular basis of this crucial aspect of HC differentiation (Jahan et al., 2013) are not profound enough to regenerate the right type of HC (Liu et al., 2014b) to ensure normal function. Defining the molecular context needed for HC type specific differentiation in conjunction with defined levels of Atoh1 expression (Jahan et al., 2013) and controlled changes of Atoh1 expression over time (Ahmed et al., 2012) will be needed to move forward.

Second, most HCs generated with Atoh1 treatment alone have limited long term viability. In part this may relate to the progressive loss of Atoh1 in these experiments that may needed to maintain long term Pou4f3 expression (Masuda et al., 2012), but in part it may also relate to an unstable transformation into $\mathrm{HC}$ that requires recapitulating the specification sequence of $\mathrm{HCs}$ precursors and their differentiation. Such critical steps might include expression of additional factors prior to and in addition to Atoh1 or the prolonged expression of critical levels of Atoh1. Human hearing loss may show partial dedifferentiation of the OC with profound local differences comparable to experimental animals (Taylor et al., 2012). A "one size fits all” approach to such heterogeneity may result in incomplete restoration.

Finally, while the single gene approach to $\mathrm{HC}$ regeneration has been extremely influential to catapult much research forward, it is now time to reflect why this approach has not lived up to its promise. We therefore suggest more complex procedures that recapitulate steps in development of the OC in addition to Atoh1. For example, expressing Eya1, Pax2, Sox2, Jag1, Foxg1, Neurod1, Neurog1, and Gata3 prior to Atoh1 expression may "prime" remaining cells of the $\mathrm{OC}$ to respond to Atoh1. Alternatively, combining Atoh1 with downstream essential genes for HC maintenance that are only partially regulated by Atoh1 (Ahmed et al., 2012), such as Pou4f3, could define the context for HC differentiation. Moreover, using transient expression of Atoh1 in already differentiated HCs might prolong their viability (Yang et al., 2012), possibly long enough to sidestep the need for OC regeneration in elderly people suffering from early stages of neurosensory hearing loss. Given the projected massive occurrence of hearing 
loss in the next 25 years, ideas revolving around maintenance of HCs using Atoh1 alone might provide more-short term benefit compared to currently impossible reconstitution of the OC after long term HC loss. Given the ability of Atoh1 to transdifferentiate supporting cells in certain conditions (Liu et al., 2014a), it might be necessary to replace Atoh 1 by other bHLH genes that can accomplish long term maintenance of HCs without risk of transforming supporting cells into HCs. We are currently working on such approaches using novel mouse models to differentiate HCs in the absence or at most transient presence of Atoh1.

\section{ACKNOWLEDGMENTS}

This work was supported by NIH (P30 DC 010362, R03 DC013655), NASA Base Program and the OVPR, University of Iowa.

\section{REFERENCES}

Ahmed, M., Wong, E. Y., Sun, J., Xu, J., Wang, F., and Xu, P.-X. (2012). Eya1-Six1 interaction is sufficient to induce hair cell fate in the cochlea by activating Atoh1 expression in cooperation with Sox2. Dev. Cell 22, 377-390. doi: 10.1016/j.devcel.2011.12.006

Ben-Arie, N., Bellen, H. J., Armstrong, D. L., McCall, A. E., Gordadze, P. R., Guo, Q., et al. (1997). Math1 is essential for genesis of cerebellar granule neurons. Nature 390, 169-172. doi: 10.1038/36579

Benito-Gonzalez, A., and Doetzlhofer, A. (2014). Hey1 and Hey2 Control the Spatial and Temporal pattern of mammalian auditory hair cell differentiation downstream of hedgehog signaling. J. Neurosci. 34, 12865-12876. doi: 10.1523/JNEUROSCI.1494-14.2014

Bermingham, N. A., Hassan, B. A., Price, S. D., Vollrath, M. A., Ben-Arie, N., Eatock, R. A., et al. (1999). Math1: an essential gene for the generation of inner ear hair cells. Science 284, 1837-1841. doi: 10.1126/science.284.5421.1837

Beurg, M., Xiong, W., Zhao, B., Müller, U., and Fettiplace, R. (2014). Subunit determination of the conductance of hair-cell mechanotransducer channels. Proc. Natl. Acad. Sci. U.S.A. doi: 10.1073/pnas.1420906112. [Epub ahead of print].

Bouchard, M., de Caprona, D., Busslinger, M., Xu, P., and Fritzsch, B. (2010). Pax2 and Pax8 cooperate in mouse inner ear morphogenesis and innervation. $B M C$ Dev. Biol. 10:89. doi: 10.1186/1471-213X-10-89

Cai, T., Seymour, M. L., Zhang, H., Pereira, F. A., and Groves, A. K. (2013), Conditional deletion of Atoh1 reveals distinct critical periods for survival and function of hair cells in the organ of Corti. J. Neurosci. 33, 10110-10122. doi: 10.1523/JNEUROSCI.5606-12.2013

Chen, P., Johnson, J. E., Zoghbi, H. Y., and Segil, N. (2002). The role of Math1 in inner ear development: uncoupling the establishment of the sensory primordium from hair cell fate determination. Development 129, 2495-2505.

Chonko, K. T., Jahan, I., Stone, J., Wright, M. C., Fujiyama, T., Hoshino, M., et al. (2013). Atoh1 directs hair cell differentiation and survival in the late embryonic mouse inner ear. Dev. Biol. 381, 401-410. doi: 10.1016/j.ydbio.2013.06.022

Dabdoub, A., Puligilla, C., Jones, J. M., Fritzsch, B., Cheah, K. S., Pevny, L. H., et al. (2008). Sox 2 signaling in prosensory domain specification and subsequent hair cell differentiation in the developing cochlea. Proc. Natl. Acad. Sci. U.S.A. 105, 18396-18401. doi: 10.1073/pnas.0808175105

Deng, M., Luo, X. J., Pan, L., Yang, H., Xie, X., Liang, G., et al. (2014). LMO4 functions as a negative regulator of sensory organ formation in the mammalian cochlea. J. Neurosci. 34, 10072-10077. doi: 10.1523/JNEUROSCI.0352-14.2014

Doetzlhofer, A., Basch, M. L., Ohyama, T., Gessler, M., Groves, A. K., and Segil, N. (2009). Hey2 regulation by FGF provides a Notch-independent mechanism for maintaining pillar cell fate in the organ of Corti. Dev. Cell 16, 58-69. doi: 10.1016/j.devcel.2008.11.008

Domínguez-Frutos, E., López-Hernández, I., Vendrell, V., Neves, J., Gallozzi, M., Gutsche, K., et al. (2011). N-myc controls proliferation, morphogenesis, and patterning of the inner ear. J. Neurosci. 31, 7178-7189. doi: 10.1523/JNEUROSCI.0785-11.2011

Driver, E. C., Sillers, L., Coate, T. M., Rose, M. F., and Kelley, M. W. (2013). The Atoh1-lineage gives rise to hair cells and supporting cells within the mammalian cochlea. Dev. Biol. 376, 86-98. doi: 10.1016/j.ydbio.2013.01.005
Duncan, J. S., and Fritzsch, B. (2013). Continued expression of GATA3 is necessary for cochlear neurosensory development. PLoS ONE 8:e62046. doi: 10.1371/journal.pone.0062046

Erkman, L., McEvilly, R. J., Luo, L., Ryan, A. K., Hooshmand, F., O'Connell, S. M., et al. (1996). Role of transcription factors Brn-3.1 and Brn-3.2 in auditory and visual system development. Nature 381, 603-606. doi: 10.1038/ $381603 \mathrm{a} 0$

Flora, A., Klisch, T. J., Schuster, G., and Zoghbi, H. Y. (2009). Deletion of Atoh1 disrupts Sonic Hedgehog signaling in the developing cerebellum and prevents medulloblastoma. Science 326, 1424-1427. doi: 10.1126/science. 1181453

Fritzsch, B., Beisel, K. W., and Hansen, L. A. (2006). The molecular basis of neurosensory cell formation in ear development: a blueprint for hair cell and sensory neuron regeneration? Bioessays 28, 1181-1193. doi: 10.1002/bies.20502

Fritzsch, B., Jahan I., Pan N., and Elliott K. L. (2015a). Evolving gene regulatory networks into cellular networks guiding adaptive behavior: an outline how single cells could have evolved into a centralized neurosensory system. Cell Tissue Res. 359, 295-313. doi: 10.1007/s00441-014-2043-1

Fritzsch, B., Matei, V. A., Nichols, D. H., Bermingham, N., Jones, K., Beisel, K. W., et al. (2005). Atoh1 null mice show directed afferent fiber growth to undifferentiated ear sensory epithelia followed by incomplete fiber retention. Dev. Dyn. 233, 570-583. doi: $10.1002 /$ dvdy.20370

Fritzsch, B., and Nichols, D. (1993). DiI reveals a prenatal arrival of efferents at the differentiating otocyst of mice. Hear. Res. 65, 51-60. doi: 10.1016/03785955(93) $90200-\mathrm{K}$

Fritzsch, B., Pan, N., Jahan, I., and Elliott, K. L. (2015b). Inner ear development: building a spiral ganglion and an organ of Corti out of unspecified ectoderm. Cell Tissue Res. doi: 10.1007/s00441-014-2031-5. [Epub ahead of print].

Giraldez, F., and Fritzsch, B. (2007). The molecular biology of ear development"Twenty years are nothing." Int. J. Dev. Biol. 51, 429. doi: 10.1387/ijdb.072390fg

Gokoffski, K. K., Wu, H. H., Beites, C. L., Kim, J., Kim, E. J., Matzuk, M. M., et al. (2011). Activin and GDF11 collaborate in feedback control of neuroepithelial stem cell proliferation and fate. Development 138, 4131-4142. doi: $10.1242 /$ dev.065870

Groves, A. K., Zhang, K. D., and Fekete, D. M. (2013). The genetics of hair cell development and regeneration. Ann. Rev. Neurosci. 36, 361-381. doi: 10.1146/annurev-neuro-062012-170309

Herculano-Houzel, S. (2009). The human brain in numbers: a linearly scaled-up primate brain. Front. Hum. Neurosci. 3:31. doi: 10.3389/neuro.09.031

Hertzano, R., Montcouquiol, M., Rashi-Elkeles, S., Elkon, R., Yucel, R., Frankel, W. N., et al. (2004). Transcription profiling of inner ears from Pou4f3(ddl/ddl) identifies Gfil as a target of the Pou4f3 deafness gene. Hum. Mol. Genet. 13, 2143-2153. doi: $10.1093 / \mathrm{hmg} / \mathrm{ddh} 218$

Imayoshi, I., and Kageyama, R. (2014). bHLH factors in self-renewal, multipotency, and fate choice of neural progenitor cells. Neuron 82, 9-23. doi: 10.1016/j.neuron.2014.03.018

Itkin-Ansari, P., Marcora, E., Geron, I., Tyrberg, B., Demeterco, C., Hao, E., et al. (2005). NeuroD1 in the endocrine pancreas: localization and dual function as an activator and repressor. Dev. Dyn. 233, 946-953. doi: 10.1002/dvdy.20443

Jahan, I., Pan, N., Kersigo, J., Calisto, L. E., Morris, K. A., Kopecky, B., et al. (2012). Expression of Neurog1 instead of Atohl can partially rescue organ of Corti cell survival. PLoS ONE 7:e30853. doi: 10.1371/journal.pone.0030853

Jahan, I., Pan, N., Kersigo, J., and Fritzsch, B. (2010). Neurod1 suppresses hair cell differentiation in ear ganglia and regulates hair cell subtype development in the cochlea. PLoS ONE 5:e11661. doi: 10.1371/journal.pone.0011661

Jahan, I., Pan, N., Kersigo, J., and Fritzsch, B. (2013). Beyond generalized hair cells: molecular cues for hair cell types. Hear. Res. 297, 30-41. doi: 10.1016/j.heares.2012.11.008

Kelly, M. C., Chang, Q., Pan, A., Lin, X., and Chen, P. (2012). Atoh1 directs the formation of sensory mosaics and induces cell proliferation in the postnatal mammalian cochlea in vivo. J. Neurosci. 32, 6699-6710. doi: 10.1523/JNEUROSCI.5420-11.2012

Kiernan, A. E., Pelling, A. L., Leung, K. K., Tang, A. S., Bell, D. M., Tease, C., et al. (2005). Sox 2 is required for sensory organ development in the mammalian inner ear. Nature 434, 1031-1035. doi: 10.1038/nature03487

Klisch, T. J., Xi, Y., Flora, A., Wang, L., Li, W., and Zoghbi, H. Y. (2011). In vivo Atoh1 targetome reveals how a proneural transcription factor regulates cerebellar development. Proc. Natl. Acad. Sci. U.S.A. 108, 3288-3293. doi: 10.1073/pnas.1100230108 
Kopecky, B., DeCook, R., and Fritzsch, B. (2012). Mutational ataxia resulting from abnormal vestibular acquisition and processing is partially compensated for. Behav. Neurosci. 126, 301. doi: 10.1037/a0026896

Kopecky, B. J., Jahan, I., and Fritzsch, B. (2013). Correct timing of proliferation and differentiation is necessary for normal inner ear development and auditory hair cell viability. Dev. Dyn. 242, 132-147. doi: 10.1002/dvdy.23910

Kopecky, B., Santi, P., Johnson, S., Schmitz, H., and Fritzsch, B. (2011). Conditional deletion of N-Myc disrupts neurosensory and non-sensory development of the ear. Dev. Dyn. 240, 1373-1390. doi: 10.1002/dvdy.22620

Lee, Y.-S., Liu, F., and Segil, N. (2006). A morphogenetic wave of p27Kip1 transcription directs cell cycle exit during organ of Corti development. Development 133, 2817-2826. doi: 10.1242/dev.02453

Li, S., Price, S. M., Cahill, H., Ryugo, D. K., Shen, M. M., and Xiang, M. (2002). Hearing loss caused by progressive degeneration of cochlear hair cells in mice deficient for the Barhll homeobox gene. Development 129, 3523-3532.

Liu, H., Pecka, J. L., Zhang, Q., Soukup, G. A., Beisel, K. W., and He, D. Z. (2014b). Characterization of transcriptomes of cochlear inner and outer hair cells. J. Neurosci. 34, 11085-11095. doi: 10.1523/JNEUROSCI.1690-14.2014

Liu, Z., Fang, J., Dearman, J., Zhang, L., and Zuo, J. (2014a). In vivo generation of immature inner hair cells in neonatal mouse cochleae by ectopic Atoh1 expression. PLoS ONE 9:e89377. doi: 10.1371/journal.pone.0089377

Ma, Q., Chen, Z., del Barco Barrantes, I., de la Pompa, J. L., and Anderson, D. J. (1998). Neurogenin 1 is essential for the determination of neuronal precursors for proximal cranial sensory ganglia. Neuron 20, 469-482. doi: 10.1016/S08966273(00)80988-5

Ma, Q., Kintner, C., and Anderson, D. J. (1996). Identification of neurogenin, a vertebrate neuronal determination gene. Cell 87, 43-52. doi: 10.1016/S00928674(00)81321-5

Mantela, J., Jiang, Z., Ylikoski, J., Fritzsch, B., Zacksenhaus, E., and Pirvola, U. (2005). The retinoblastoma gene pathway regulates the postmitotic state of hair cells of the mouse inner ear. Development 132, 2377-2388. doi: 10.1242/dev.01834

Masuda, M., Pak, K., Chavez, E., and Ryan, A. F. (2012). TFE2 and GATA3 enhance induction of POU4F3 and myosin VIIa positive cells in nonsensory cochlear epithelium by ATOH1. Dev. Biol. 372, 68-80. doi: 10.1016/j.ydbio.2012.09.002

Matei, V., Pauley, S., Kaing, S., Rowitch, D., Beisel, K. W., Morris, K., et al. (2005). Smaller inner ear sensory epithelia in Neurog 1 null mice are related to earlier hair cell cycle exit. Dev. Dyn. 234, 633-650. doi: 10.1002/dvdy.20551

Mulvaney, J., and Dabdoub, A. (2012). Atoh1, an essential transcription factor in neurogenesis and intestinal and inner ear development: function, regulation, and context dependency. J. Assoc. Res. Otolaryngol. 13, 281-293. doi: 10.1007/s10162-012-0317-4

Nichols, D. H., Pauley, S., Jahan, I., Beisel, K. W., Millen, K. J., and Fritzsch, B. (2008). Lmxla is required for segregation of sensory epithelia and normal ear histogenesis and morphogenesis. Cell Tissue Res. 334, 339-358. doi: 10.1007/s00441-008-0709-2

Ono, K., Kita, T., Sato, S., O’Neill, P., Mak, S.-S., Paschaki, M., et al. (2014). FGFR1-Frs2/3 signalling maintains sensory progenitors during inner ear hair cell formation. PLoS Genet. 10:e1004118. doi: 10.1371/journal.pgen. 1004118

Pan, N., Jahan, I., Kersigo, J., Duncan, J. S., Kopecky, B., and Fritzsch, B. (2012). A novel Atoh1 "self-terminating" mouse model reveals the necessity of proper Atoh1 level and duration for hair cell differentiation and viability. PLoS ONE 7:e30358. doi: 10.1371/journal.pone.0030358

Pan, N., Jahan, I., Kersigo, J., Kopecky, B., Santi, P., Johnson, S., et al. (2011). Conditional deletion of Atoh1 using Pax2-Cre results in viable mice without differentiated cochlear hair cells that have lost most of the organ of Corti. Hear. Res. 275, 66-80. doi: 10.1016/j.heares.2010.12.002

Pan, N., Jahan, I., Lee, J. E., and Fritzsch, B. (2009). Defects in the cerebella of conditional Neurod1 null mice correlate with effective $\operatorname{Tg}$ (Atoh1-cre) recombination and granule cell requirements for Neurod1 for differentiation. Cell Tissue Res. 337, 407-428. doi: 10.1007/s00441-009-0826-6

Pan, W., Jin, Y., Chen, J., Rottier, R. J., Steel, K. P., and Kiernan, A. E. (2013). Ectopic expression of activated notch or SOX2 reveals similar and unique roles in the development of the sensory cell progenitors in the mammalian inner ear. J. Neurosci. 33, 16146-16157. doi: 10.1523/JNEUROSCI.315012.2013
Pan, W., Jin, Y., Stanger, B., and Kiernan, A. E. (2010). Notch signaling is required for the generation of hair cells and supporting cells in the mammalian inner ear. Proc. Natl. Acad. Sci. U.S.A. 107, 15798-15803. doi: 10.1073/pnas.10030 89107

Pauley, S., Kopecky, B., Beisel, K., Soukup, G., and Fritzsch, B. (2008). Stem cells and molecular strategies to restore hearing. Panminerva Med. 50, 41-53.

Reiprich, S., and Wegner, M. (2014). From CNS stem cells to neurons and glia: sox for everyone. Cell Tissue Res. 359, 111-124. doi: 10.1007/100441-014-1909-6

Rocha-Sanchez, S. M., Scheetz, L. R., Contreras, M., Weston, M. D., Korte, M., McGee, J., et al. (2011). Mature mice lacking Rbl2/p130 gene have supernumerary inner ear hair cells and supporting cells. J. Neurosci. 31, 8883-8893. doi: 10.1523/JNEUROSCI.5821-10.2011

Ruben, R. J. (1967). Development of the inner ear of the mouse: a radioautographic study of terminal mitoses. Acta Otolaryngol. (Suppl. 220), 1-44.

Schimmang, T. (2013). Transcription factors that control inner ear development and their potential for transdifferentiation and reprogramming. Hear. Res. 297, 84-90. doi: 10.1016/j.heares.2012.11.001

Schimmang, T., and Pirvola, U. (2013). Coupling the cell cycle to development and regeneration of the inner ear. Semin. Cell Dev. Biol. 24, 507-513. doi: 10.1016/j.semcdb.2013.04.004

Sheykholeslami, K., Thimmappa, V., Nava, C., Bai, X., Yu, H., Zheng, T., et al. (2013). A new mutation of the Atoh1 gene in mice with normal life span allows analysis of inner ear and cerebellar phenotype in aging. PLoS ONE 8:e79791. doi: 10.1371/journal.pone.0079791

Taylor, R. R., Jagger, D. J., and Forge, A. (2012). Defining the cellular environment in the organ of Corti following extensive hair cell loss: a basis for future sensory cell replacement in the cochlea. PLoS ONE 7:e30577. doi: 10.1371/journal.pone.0030577

Vahava, O., Morell, R., Lynch, E. D., Weiss, S., Kagan, M. E., Ahituv, N., et al. (1998). Mutation in transcription factor POU4F3 associated with inherited progressive hearing loss in humans. Science 279, 1950-1954. doi: 10.1126/science.279.5358.1950

Woods, C., Montcouquiol, M., and Kelley, M. W. (2004). Mathl regulates development of the sensory epithelium in the mammalian cochlea. Nat. Neurosci. 7 , 1310-1318. doi: 10.1038/nn1349

Xiang, M., Maklad, A., Pirvola, U., and Fritzsch, B. (2003). Brn3c null mutant mice show long-term, incomplete retention of some afferent inner ear innervation. BMC Neurosci. 4:2. doi: 10.1186/1471-2202-4-2

Yang, S. M., Chen, W., Guo, W. W., Jia, S., Sun, J. H., Liu, H. Z., et al. (2012). Regeneration of stereocilia of hair cells by forced Atohl expression in the adult mammalian cochlea. PLoS ONE 7:e46355. doi: 10.1371/journal.pone.0046355

Zine, A., Aubert, A., Qiu, J., Therianos, S., Guillemot, F., Kageyama, R., et al. (2001). Hes 1 and Hes5 activities are required for the normal development of the hair cells in the mammalian inner ear. J. Neurosci. 21, 4712-4720.

Zine, A., Löwenheim, H., and Fritzsch, B. (2014). Toward Translating Molecular Ear Development to Generate Hair Cells from Stem Cells, Adult Stem Cells. New York, NY: Springer, 111-161.

Zou, D., Silvius, D., Fritzsch, B., and Xu, P.-X. (2004). Eyal and Sixl are essential for early steps of sensory neurogenesis in mammalian cranial placodes. Development 131, 5561-5572. doi: 10.1242/dev.01437

Conflict of Interest Statement: The authors declare that the research was conducted in the absence of any commercial or financial relationships that could be construed as a potential conflict of interest.

Received: 24 November 2014; accepted: 14 January 2015; published online: 05 February 2015.

Citation: Jahan I, Pan N and Fritzsch B (2015) Opportunities and limits of the one gene approach: the ability of Atoh1 to differentiate and maintain hair cells depends on the molecular context. Front. Cell. Neurosci. 9:26. doi: 10.3389/fncel.2015.00026 This article was submitted to the journal Frontiers in Cellular Neuroscience.

Copyright (C) 2015 Jahan, Pan and Fritzsch. This is an open-access article distributed under the terms of the Creative Commons Attribution License (CC BY). The use, distribution or reproduction in other forums is permitted, provided the original author(s) or licensor are credited and that the original publication in this journal is cited, in accordance with accepted academic practice. No use, distribution or reproduction is permitted which does not comply with these terms. 